\title{
Correlation of photophysical parameters with macrocycle distortion in porphyrins with graded degree of saddle distortion
}

\author{
Beate Röder, ${ }^{* a}$ Maren Büchner, ${ }^{a}$ Ilja Rückmann ${ }^{a}$ and Mathias O. Senge ${ }^{* b}$ \\ Received 30th April 2010, Accepted 4th June 2010 \\ First published as an Advance Article on the web 23rd June 2010 \\ DOI: 10.1039/c0pp00107d
}

Different porphyrin conformations are believed to play a role in controlling the cofactor properties in natural tetrapyrrole-protein complexes. In order to study the correlation between macrocycle nonplanarity and physicochemical properties in detail, a series of six porphyrins with graded degree of macrocycle distortion was investigated. These conformationally designed porphyrins are based on the successive introduction of $\beta$-ethyl groups into the tetraphenylporphyrin parent macrocycle and the degree of nonplanarity is dependent on the number and localization of the $\beta$-ethyl meso-phenyl interactions. The electronic properties of the complete series of porphyrins were investigated in solution. It was found that the singlet and triplet properties depend not only on the out-of-plane distortion parameter but also on the type of central metal. Moreover, it was found that macrocycle distortion affects the singlet state properties significantly stronger than the triplet properties. In addition, the efficiency of energy transfer to molecular oxygen was investigated. It was shown that the singlet oxygen quantum yield depends strongly on the triplet state lifetime of the porphyrins, resulting in differences between the decrease of intersystem crossing and singlet oxygen quantum yield. The observed gradual change of electronic parameters of base free tetraphenylporphyrins with increasing deformation of the macrocycle indicates the validity of using conformationally designed porphyrins to fine-tune photophysical properties.

\section{Introduction}

Porphyrins are ubiquitous cofactors in nature and function in a wide range of biological processes ranging from respiration, electron transfer, oxidation catalysis, signaling to photosynthesis. ${ }^{1}$ Despite the wide variety of chemically catalyzed reactions only a small number of tetrapyrrole cofactors - mostly heme and chlorophyll derivatives - are involved in these reactions. In addition to their basic macrocycle properties, i.e., coordination properties and functional groups their conformation is of significant relevance for the biological function. ${ }^{2,3}$ This is based on the hypothesis that fine-tuning of the macrocycle conformation by the protein scaffold is one way by which nature might control the physicochemical properties of the cofactors in intact tetrapyrroleprotein complexes. ${ }^{2-5}$ This can account for the often quite different functions of the same chromophore when bound to different apoproteins. As a result porphyrins with nonplanar macrocycle conformations have attracted considerable attention. ${ }^{6-8}$ Indeed, more and more nonplanar tetrapyrrole macrocycle conformations have been identified in porphyrin protein complexes, in fact more cases than can be listed here. ${ }^{3,9}$ Suitable biomimetic compounds typically comprise highly substituted porphyrins with nonplanar conformations where steric congestion at the porphyrin periphery results in distorted macrocycles. ${ }^{10-12}$

${ }^{a}$ Humboldt-Universität zu Berlin, Institut für Physik, AG Photobiophysik, Newtonstr. 15, 12489, Berlin, Germany.E-mail: beate.roeder@physik. hu-berlin.de; Fax: +49 302093 7666; Tel: +49 3020937625

${ }^{b}$ School of Chemistry, SFI Tetrapyrrole Laboratory, Trinity College Dublin, Dublin 2, Ireland.E-mail: sengem@tcd.ie; Fax: +35318968536; Tel: +353 18968537
A considerable body of information has now accumulated for these conformationally strained porphyrins. Amongst many, ${ }^{7}$ one prominent example is the group of $2,3,7,8,12,13,17,18$-octaalkyl$5,10,15,20$-tetraarylporphyrins. ${ }^{6,13}$ Due to their symmetry, they are easily prepared and in most cases exhibit highly nonplanar saddle-shaped macrocycle conformations. Such compounds serve as biomimetic compounds for conformational effects found in natural in vivo systems where the steric effects of the apoprotein on the cofactor are mimicked through chemical manipulation and substituent effects on the macrocycle (Fig. 1).

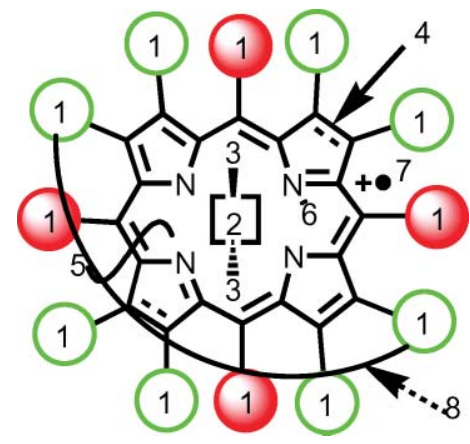

Fig. 1 Illustration of the chemical possibilities for altering the conformation of porphyrins in biomimetic compounds. (1) Introduction of sterically demanding substituents; (2) metallation; (3) axial ligands; (4) degree of reduction; (5) interruption of the conjugated system; (6) protonation, alkylation; (7) radical cations, aggregation and packing effects; (8) strapping. Figure modified from ref. 7.

Here, 2,3,7,8,12,13,17,18-octaethyl-5,10,15,20-tetraphenylporphyrin (OETPP), the structural hybrid of the planar 
octaethylporphyrin (OEP) and tetraphenylporphyrin (TPP), has become a standard nonplanar porphyrin of choice and has been used in many physical, theoretical and spectroscopic studies on porphyrin nonplanarity. ${ }^{6,13,14}$

However, the use of these highly nonplanar porphyrins as biomimetics can be problematic. Naturally occurring dyes have asymmetric substituent pattern and show distortions smaller than those observed in highly nonplanar dodecasubstituted porphyrins. Likewise, many physicochemical investigations on nonplanar porphyrins have studied all-or-nothing effects, e.g., by comparison of a planar porphyrin such as TPP or OEP with a very nonplanar porphyrin like OETPP. ${ }^{15}$ Thus, the interpretation of physicochemical data and more insight into their biological function requires the use of rationally planned series of closely related porphyrins with different degree of macrocycle distortion.

One such series has been developed by us earlier during a comprehensive study of asymmetrically substituted porphyrins. In light of the wealth of structural and physicochemical data available for the sterically unhindered TPP and OEP and now for the nonplanar OETPP, a porphyrin series of choice was prepared via synthesis of ethyl-substituted tetraphenylporphyrins through mixed condensation of pyrrole, diethylpyrrole and benzaldehyde. ${ }^{16}$ The formal introduction of successively more $\beta$-ethyl groups into the tetraphenylporphyrin parent macrocycle gave access to TPP, diethyltetraphenylporphyrin (DETPP), two regioisomers of tetraethyltetraphenylporphyrin (tTETPP, cTETPP), hexaethyltetraphenylporphyrin (HETPP) and OETPP (Fig. 2). It thus provided a series of six closely related porphyrins providing enough data points for a more detailed analysis which proved useful for physical, ${ }^{17}$ structural ${ }^{18}$ and chemical ${ }^{19}$ investigations.

Despite this interest, the photophysical properties of such a closely related series of nonplanar porphyrins with graded degree of steric strain have not been investigated in detail. Since most of the natural function of, for example, the chlorophylls is related to their photophysical properties such studies are mandatory. Since we reported on the static steady state electronic absorption properties, ${ }^{16}$ some more detailed information about the photophysical properties of these compounds has become available. ${ }^{20,21}$ However, these studies addressed only individual members of the series of six porphyrins and did not provide any clear cut information on the gradual changes in the photophysical parameters of these nonplanar porphyrins. These studies presented selected properties only and did not give a full set of electronic parameters after light absorption. Thus, we present here a detailed photophysical investigation of singlet and triplet parameters of the members of this series and compare them with the relevant data from the literature.

\section{Results and discussion}

\section{Structural analysis}

A detailed description of all these structures would take too much space and the reader is referred to the original articles ${ }^{16}$ for complete listings of all conformational and geometrical parameters and their comparison with the relevant TPP, OEP and OETPP structures. Suffice it to say that the basic premise, i.e. increasing numbers of peripheral interactions lead to increasing nonplanar conformations could be proven unambiguously. As shown in Fig. 2

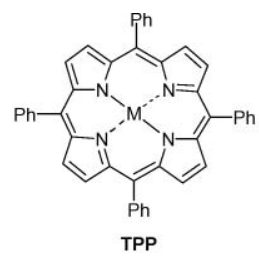

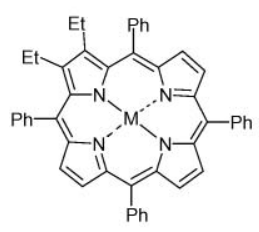
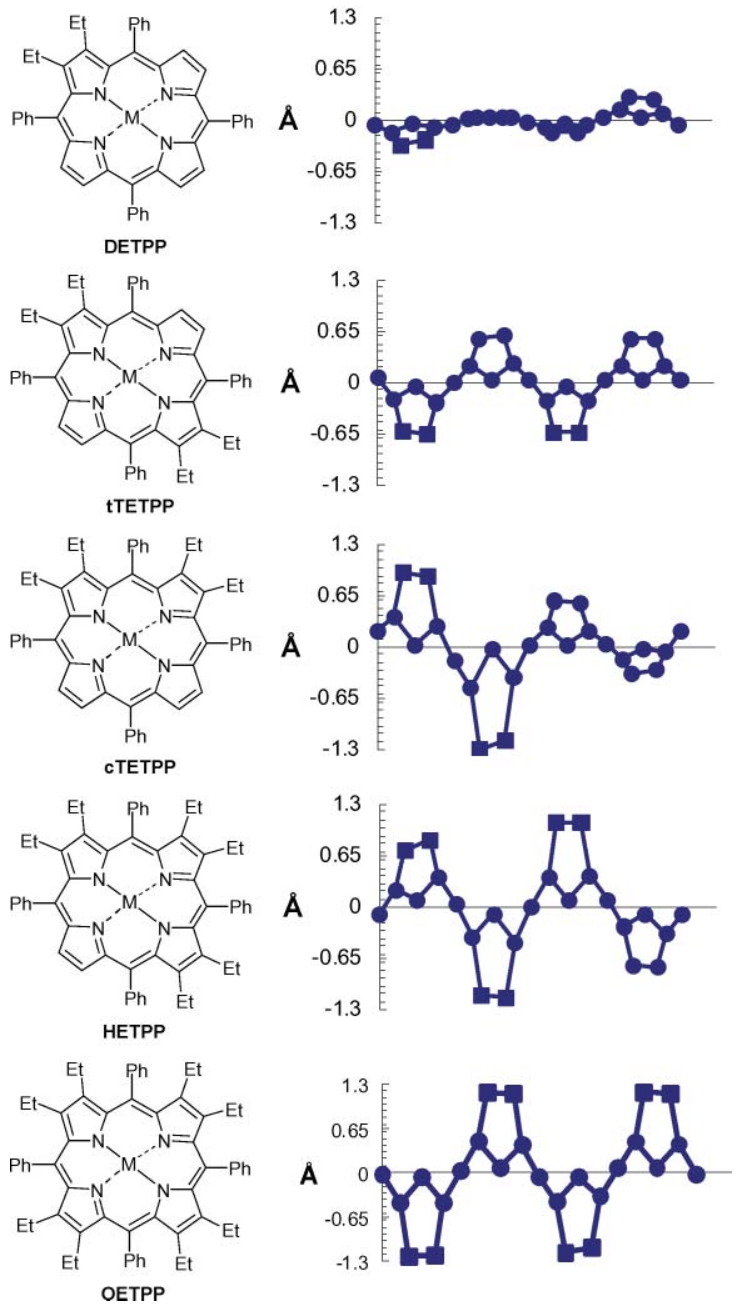

Fig. 2 Molecular structures of the compounds studied. The right panels shows the skeletal deviations of the macrocycle atoms from the $4 \mathrm{~N}$-plane in the crystal structures of the respective free base porphyrins. Indicates the $\beta$-carbon atoms bearing ethyl substituents.

for the free base porphyrins, the degree of nonplanarity increases in the order: TPP $<$ DETPP $<$ tTETPP $<$ cTETPP $<$ HETPP $<$ OETPP, i.e. with increasing degree of $\beta$-ethyl substitution and the number and localization of potential $\beta$-ethyl meso-phenyl interactions. This holds true for the free bases and the related metal complexes and chemical derivatives thereof. ${ }^{16,18,19}$ The data correlate with increasing bathochromic shifts of the absorption bands in solution which is a key characteristic of sad distorted porphyrins. ${ }^{4,7,22}$

Depending on the substituent pattern, saddle-shaped macrocycle conformations of different degrees of distortion were observed (Fig. 2). While the conformation of tTETPP is symmetric, DETPP, cTETPP and HETPP show asymmetric distortion modes with individual $\beta$-pyrrole displacements reaching those described for 
Table 1 Average deviation of the 24 macrocycle atoms from their leastsquares-plane

\begin{tabular}{lll}
\hline Porphyrin & Molecular weight $/ \mathrm{g} \mathrm{mol}^{-1}$ & $\Delta 24[\AA]^{a}$ \\
\hline $\mathrm{H}_{2}$ TPP & 614.76 & 0.05 \\
$\mathrm{H}_{2}$ DETPP & 670.84 & 0.10 \\
$\mathrm{H}_{2}$ tTETPP & 726.95 & 0.29 \\
$\mathrm{H}_{2}$ CTETPP & 726.95 & 0.38 \\
$\mathrm{H}_{2}$ HETPP & 783.05 & 0.46 \\
$\mathrm{H}_{2}$ OETPP & 839.16 & 0.54 \\
$\mathrm{ZnTPP}$ & 678.12 & 0.05 \\
$\mathrm{Zn}(\mathrm{II})$ DETPP & 734.22 & 0.18 \\
Zn(II)tTETPP & 790.33 & 0.28 \\
Zn(II)cTETPP & 790.33 & 0.33 \\
Zn(II)HETPP & 846.43 & 0.43 \\
Zn(II)OETPP & 902.53 & 0.48
\end{tabular}

${ }^{a} \Delta 24$ is the average deviation of the 24 macrocycle atoms from their leastsquares plane.

dodecasubstituted porphyrins. Overall, higher displacements from planarity were found close to $\beta$-ethyl-meso-phenyl groups while smaller displacements were observed in parts of the molecules bearing $\beta$-hydrogen atoms. ${ }^{16}$ As observed for symmetric, nonplanar porphyrins, larger metals led to a decrease in conformational distortion with associated changes in bond lengths and bond angles. Note, that significant degrees of ruffling can be observed and appear to be larger in more asymmetrically substituted porphyrins. Relevant structural data are compiled in Table 1.

\section{Steady state absorption and fluorescence spectra}

The absorption spectra of the compounds (XETPP) show an increasing red shift of the Soret-band with increasing distortion (not shown). The free base porphyrin spectra have the well known 4 Q-band structure in the red region (Fig. 3), whereas for the Zn-porphyrins two Q-bands were observed (not shown).

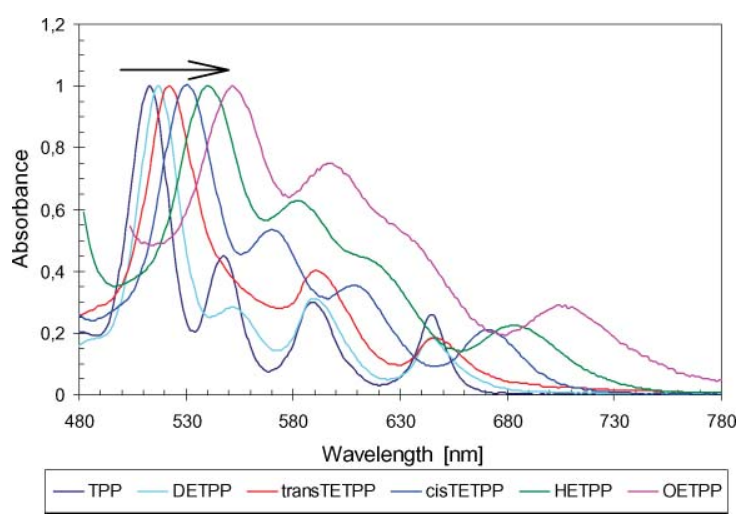

Fig. 3 Normalized absorption spectra of the free base porphyrins in the Q-Band region in DMF.

As shown in Fig. 3, the relative absorbance in the Q-band region changes with increasing distortion. In addition to the bathochromic shift, relative intensity changes and broadening of the bands were observed as well. This behavior already gives an indication to relevant differences of related electronic parameters.
The differences in the Q-band absorption are also reflected in the spectral shape of fluorescence (Fig. 4). Whereas the porphyrins TPP, DETPP and TTETPP show the typical twopeak fluorescence the spectral shape dramatically changes for compounds with higher distortion. Besides the already mentioned bathochromic shift, the spectra lose more and more their structure with increasing macrocycle distortion. For OETPP only one broad fluorescence band was observed.

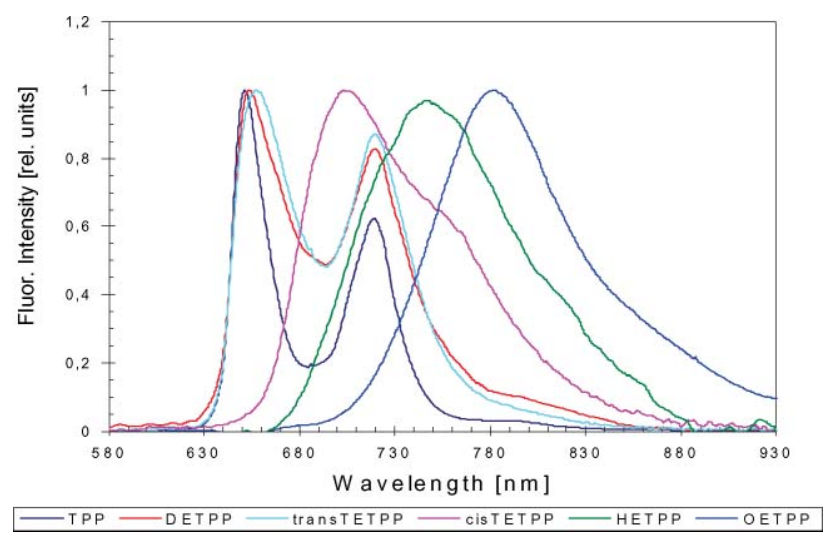

Fig. 4 Fluorescence spectra of the free base porphyrins in DMF $\left(\lambda_{\text {exc }}=\right.$ $415 \mathrm{~nm} ; \mathrm{OD}=0.1$ at $415 \mathrm{~nm}$ ).

The Stokes-shift of the $\mathrm{Zn}$ (II) and free base porphyrins is shown in Fig. 5. Besides the influence of distortion, it illustrates the different influence of $\mathrm{Zn}$ (II) and $\mathrm{H}_{2}$ on the $\mathrm{S}_{0} \mathrm{~S}_{1}$ transition. Whereas the Stokes shift for free base porphyrins starts to change only with larger distortion (cTETPP), it changes for the Zn-XETPPs at very small distortion [Zn(II)DETPP]. Moreover for the $\mathrm{Zn}$ (II) complexes, it very quickly reaches the maximum [Zn(II)cTETPP] whereas the Stokes shift of the free base XETPPs is approached only with the largest distortion. Its value is comparable to that of the $\mathrm{Zn}(\mathrm{II}) \mathrm{XETPPs}$ but is still smaller.

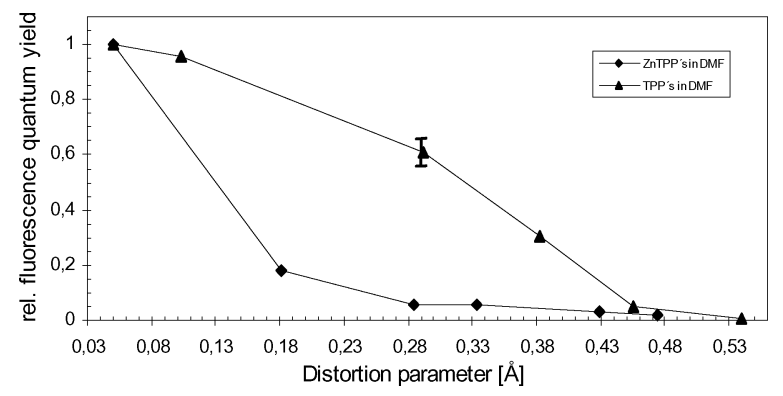

Fig. 5 Stokes shift of the porphyrins solved in DMF ( $\bullet$ : Zn(II) porphyrins; $\boldsymbol{\Lambda}$ : free base porphyrins).

\section{Fluorescence quantum yields}

Not only the shape and spectral distribution of fluorescence change, but also the fluorescence quantum yield decreases with preceding distortion. This tendency is in agreement with the results of Barkigia et al. ${ }^{6}$ For both Zn(II)- and free base OETPP it reaches less then $10 \%$ of the value observed for TPP (Fig. 6).

Again a different behavior of the Zn(II) and free base XETPPs was observed with increasing distortion. Whereas the fluorescence 


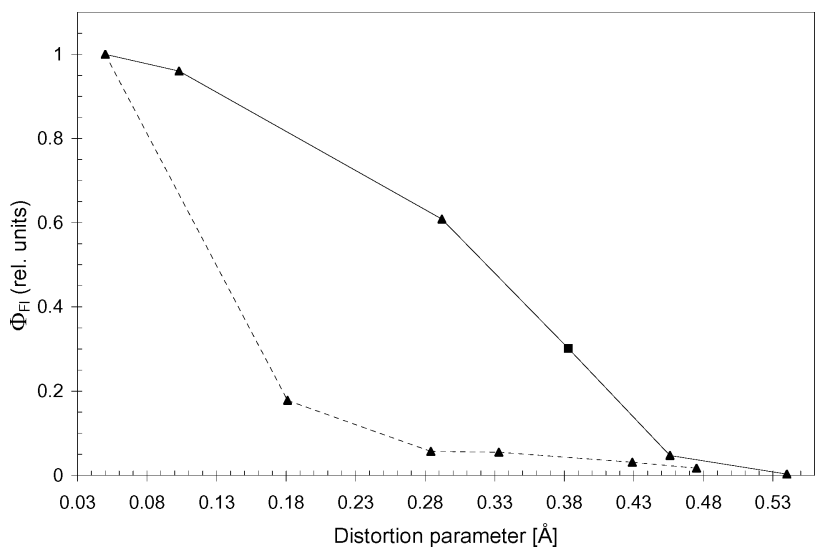

Fig. 6 Correlation between the relative fluorescence quantum yield $\left(\Phi_{\mathrm{F} 1}\right)$ and macrocycle distortion (dashed line: $\mathrm{Zn}$ (II) porphyrins; full line: free base porphyrins), all measurements in DMF.

of the $\mathrm{Zn}$-XETPPs breaks down with low distortion, for the free base compounds a gradual decrease was observed.

These findings demonstrate the strong influence of the central metal atom on the electronic properties of distorted porphyrins. The gradual change of electronic properties makes the free base XETPPs very interesting for further use since it suggests the possibility of stepwise property tuning.

\section{Fluorescence decay}

In order to make sure that no other process than distortion causes the strong decrease of fluorescence quantum yield, the fluorescence decay was investigated and the fluorescence lifetimes were calculated (Table 2).

All decay curves were successfully fitted mono-exponentially. The obtained values reflect the well known behavior of ten-fold longer decay times of free base TPPs compared to $\mathrm{Zn}$ (II)-TPPs. ${ }^{23}$ Also the correlation between decreasing quantum yield and shorter lifetime of the first excited singlet state can clearly be seen. In ref. 20 the authors report about a comparative study on basic electronic parameters of free base cTEPP, HETPP and OETPP in three solvents of different polarity: acetonitrile, $n$-hexane and toluene. They report a two exponential decay for each of the compounds independent on the solvent. During our experiments on the series of XETPP we found that the compounds are not very photostable in toluene and after a short illumination time equilibrium of two species is reached. The kinetics of this process can be followed in the steady-state absorption spectra: an isosbestic point exists and linear behavior of the curves in the extinction coefficient difference diagram was obtained (not shown). This

Table $2 \mathrm{~S}_{1}$-lifetimes determined via TCSPC in DMF. The optical density was 0.2 at the excitation wavelength of $425 \mathrm{~nm}$

\begin{tabular}{lll}
\hline & \multicolumn{2}{l}{$\tau_{\mathrm{s} 1} / \mathrm{ns}$} \\
\cline { 2 - 3 } Compound & $\mathrm{H}_{2}$ & $\mathrm{Zn}$ \\
\hline TPP & $10.6 \pm 0.3$ & $1.96 \pm 0.1$ \\
DETPP & $7.8 \pm 0.3$ & $0.9 \pm 0.2$ \\
tTETPP & $4 \pm 0.3$ & $0.4 \pm 0.1$ \\
cTETPP & $1.5 \pm 0.1$ & $0.2 \pm 0.1$ \\
HETPP & $1.4 \pm 0.2$ & 0.140 .1 \\
OETPP & $1.1 \pm 0.2$ & $0.12 \pm 0.1$ \\
\hline
\end{tabular}

fact could explain the two-exponential decay described in ref. 20. Our studies were performed in DMF because of the much higher photostability of the compounds and may give a rationale for the observed strongly mono-exponential fluorescence decay.

\section{Triplet $\left(T_{1}\right)$ state properties}

Besides the properties of the ground and first excited singlet states the population and depopulation of the first excited triplet state were investigated. Due to its long lifetime this electronic state is of particular interest. The intersystem crossing quantum yield $\left(\Phi_{\text {ISC }}\right)$ and singlet oxygen quantum yield $\left(\Phi_{\Delta}\right)$ were obtained from ps-TAS and luminescence measurements, respectively, and the phosphorescence spectra were recorded.

\section{ISC quantum yields}

The tendency of changes of electronic parameters in dependence on the distortion found for the singlet states parameters could be confirmed for the ISC quantum yield $\left(\Phi_{\text {ISC }}\right)$, too. A gradual decrease of $\Phi_{\text {ISC }}$ was observed for the free base XETPPs (Table 3). In contrast, the $\Phi_{\text {ISC }}$ of Zn-XETPPs breaks down for ZnHETPP and rises up again for $\mathrm{ZnOETPP}$.

In distinction to the observed dramatic decrease of $\Phi_{\mathrm{Fl}}$ for all compounds to less then $10 \%$ of TPP, the triplet quantum yield decreases only to about $50 \%$ compared to TPP. This opens interesting questions, especially related to energy transfer from the excited triplet state to molecular oxygen.

\section{Singlet oxygen quantum yield}

The values of singlet oxygen quantum yield $\left(\Phi_{\Delta}\right)$ of all compounds are given in Table 4. As can be seen the ability of the XETPPs to transfer energy to molecular oxygen decreases with increasing distortion, too.

Table 3 ISC quantum yields of the compounds in DMF

\begin{tabular}{lll}
\hline & \multicolumn{1}{l}{$\Phi_{\text {ISC }}$} \\
\cline { 2 - 3 } Compound & $\mathrm{H}_{2}$ & $\mathrm{Zn}$ \\
\hline TPP & $0.72 \pm 0.05$ & $0.93 \pm 0.05$ \\
DETPP & $0.68 \pm 0.05$ & $0.67 \pm 0.1$ \\
tTETPP & $0.63 \pm 0.05$ & $0.45 \pm 0.1$ \\
cTETPP & $0.42 \pm 0.05$ & $0.62 \pm 0.1$ \\
HETPP & $0.32 \pm 0.05$ & - \\
OETPP & $0.35 \pm 0.1$ & $0.4 \pm 0.1$ \\
\hline
\end{tabular}

Table 4 Singlet oxygen quantum yields in DMF. The optical density was 0.2 at the excitation wavelength of $514 \mathrm{~nm}$

\begin{tabular}{llllll}
\hline & \multicolumn{2}{l}{$\Phi_{\Delta}($ rel $)$} & & & \multicolumn{2}{c}{$\Phi_{\Delta}(\mathrm{abs} .)^{a}$} & \\
\cline { 2 - 3 } \cline { 5 - 6 } Compound & $\mathrm{H}_{2}$ & $\mathrm{Zn}$ & & $\mathrm{H}_{2}$ & $\mathrm{Zn}$ \\
\hline TPP & 1 & 1 & 0.65 & 0.75 \\
DETPP & 0.94 & 0.81 & 0.61 & 0.61 \\
tTETPP & 0.83 & 0.45 & 0.54 & 0.34 \\
cTETPP & 0.13 & 0.12 & 0.08 & 0.09 \\
HETPP & 0.11 & - & 0.07 & - \\
OETPP & 0.32 & 0.24 & 0.2 & 0.18
\end{tabular}

${ }^{a}$ The value of $\mathrm{H}_{2}$ TPP which is similar $( \pm 5 \%)$ in all organic solvents was used as reference. ${ }^{24}$ 
Table 5 Spectral position of the phosphorescence maxima in MTHF measured at $80 \mathrm{~K}$. The optical density was 0.2 at the excitation wavelength of $514 \mathrm{~nm}$

\begin{tabular}{lll}
\hline Compound & maxima/nm $(\mathrm{eV})$ & \\
\hline $\mathrm{H}_{2}$ TPP & $866(1.43)$ & $983(1.26)$ \\
$\mathrm{H}_{2}$ DETPP & $966(1.28)$ & $1086(1.14)$ \\
$\mathrm{H}_{2}$ transTETPP & $925(1.34)$ & $1060(1.22)$ \\
$\mathrm{H}_{2}$ cisTETPP & $893(1.39)$ & $1020(1.22)$ \\
$\mathrm{H}_{2}$ OETPP & $1000(1.24)$ & $1099(1.13)$ \\
\hline
\end{tabular}

However, in detail the obtained $\Phi_{\Delta}$ values do not reflect the overall tendency of the triplet quantum yields decrease. Interestingly, for tTETPP with $\Phi_{\text {ISC }}=0.63$, the $\Phi_{\Delta}$ is quite high: 0.54 . The "cis" analogue shows a very different behavior: with $\Phi_{\text {ISC }}=0.42$, its $\Phi_{\Delta}$ is only of about 0.1 . A similar behavior was observed for HETPP: $\Phi_{\text {ISC }}=0.32$ and $\Phi_{\Delta}$ is less then 0.1 .

This might be related to the conformation of the XETPPs in not allowing efficient contact with molecular oxygen. Another more probable explanation could be that the energetic position of the triplet state precludes energy transfer to molecular oxygen. To answer this question phosphorescence measurements on the free base XETPPs were carried out.

\section{Phosphorescence}

In order to avoid the quenching of the triplet state by molecular oxygen to gain analyzable phosphorescence spectra the metal free XETPPs were solved in methyltetrahydrofuran (MTHF) and frozen. MTHF was used because of its ability to form a transparent glass after freezing. All spectra (not shown) have two maxima listed in Table 5.

For the activation of singlet oxygen energy of about $0.96 \mathrm{eV}$ is needed. As can be seen, all triplet states fit this requirement. Surprisingly the DETPP triplet state has an even higher energy then that of cTETPP. In the first case appropriate singlet oxygen generation was observed, in the second case not. Moreover, comparing t- with cTETPP, it can be concluded that the energetic position of the first excited triplet state cannot be the reason for the strong decrease of the singlet oxygen quantum yield observed for cTETPP. Moreover the singlet oxygen quantum yield increases for both $\mathrm{Zn}$ - and free base OETPP, the compounds with the highest distortion. Although the absolute $\Phi_{\Delta}$ is not very high, the singlet oxygen generation efficiency increases related to $\Phi_{\text {ISC }}$.

Another possible reason for the discrepancy between the decrease of $\Phi_{\text {ISC }}$ and $\Phi_{\Delta}$ with increasing distortion could be different triplet decay kinetics. To answer this question the triplet lifetime of the XETPPs was investigated.

\section{Triplet parameters}

Beside triplet quantum yield also the triplet lifetime influences the singlet oxygen quantum yield, since: $\Phi_{\Delta} \sim \Phi_{\mathrm{ISC}} \tau_{\mathrm{T}}$.

To evaluate the influence of triplet lifetime on $\Phi_{\Delta}$ laser flash photolysis experiments were carried out under normal and oxygen reduced conditions. In Table 6 the values obtained for triplet quantum yields and lifetimes are summarized.

From the relations $1 / \tau_{\mathrm{o}}=k_{\mathrm{Ph}}$ and $1 / \tau_{\mathrm{m}}=k_{\mathrm{Ph}}+k_{\mathrm{SS}}$ follows: $1 / \tau_{\mathrm{m}}-1 / \tau_{\mathrm{o}}=k_{\mathrm{SS}}$. Using the equation $k_{\mathrm{SS}} \tau_{\mathrm{m}}=\Phi^{\prime}$, the quantum yield of triplet quenching by molecular oxygen can be calculated. With the known absolute singlet oxygen quantum yield now $\Phi_{\text {ISC }}$ can be calculated:

$$
\frac{1}{\Phi^{\prime}} \Phi_{\Delta}(\mathrm{abs})=\Phi_{\mathrm{ISC}}
$$

As can be seen from Table 6, the calculated values of triplet quantum yields $\left[\Phi_{\text {ISC }}(\right.$ calc.) $]$ are in good agreement with those obtained from ps-TAS measurements (see Table 3).

For TPP the triplet lifetime under normal and oxygen-reduced conditions differs for more then four orders of magnitude. This is reflected by a very effective singlet oxygen generation (see Table 3). The difference between these lifetimes decreases already for DETPP for one order of magnitude.

Comparing the triplet lifetime under oxygen reduced conditions $\left(\tau_{\mathrm{o}}\right)$ for the c- and tTETPP the reason for the dramatically reduced singlet oxygen quantum yield of cTETPP becomes clear: It is reduced from $21.7 \mu$ s ("trans") to $0.42 \mu$ s ("cis") for the free base and from $20 \mu$ s ("trans") to $1.41 \mu$ s ("cis") for the $\mathrm{Zn}$ (II) analogues. This result demonstrates that the low $\Phi_{\Delta}$ is caused by dramatically decreased triplet lifetimes, which results from the specific conformation of the cTETPP.

In contrast to ref. 21 we did not observe triplet decay curves for cTETPP and HETPP containing three components. Since toluene was used in ref. 21 the above mentioned photodegradation could be the reason for this behavior. Moreover, the comparative study of c- and tTETPP given here allows a new interpretation of the different triplet decay times observed in ref. 21.

Again OTEPP shows a single behavior. The triplet lifetime under normal conditions is very short $\left(\mathrm{H}_{2}: 5.4 \mu \mathrm{s} ; \mathrm{Zn}\right.$ (II): $\left.1.88 \mu \mathrm{s}\right)$ and shortens only slightly under reduced oxygen conditions $\left(\mathrm{H}_{2}: 1.1\right.$ $\mu \mathrm{s} ; \mathrm{Zn}(\mathrm{II}): 0.5 \mu \mathrm{s})$. However the triplet quenching constant $\left(k_{\mathrm{Ss}}\right)$ and $\Phi^{\prime}$ are higher then for cTETPP at even nearly the same $\Phi_{\text {ISC }}$.

Table 6 Triplet lifetimes under normal and oxygen reduced conditions in DMF. The optical density was 0.2 at the excitation wavelength. $\tau_{\mathrm{m}}$ : triplet lifetime under normal conditions; $\tau_{\mathrm{o}}$ : triplet lifetime under nitrogen bubbling; $k_{\mathrm{ss}}$ : triplet quenching constant under oxygen; $\Phi^{\prime}$ : quantum yield of triplet deactivation by molecular oxygen; $\lambda_{\text {test }}$ : wavelength of the test pulse; $\lambda_{\text {exc }}$ : wavelength of the pump pulse

\begin{tabular}{|c|c|c|c|c|c|c|c|c|c|c|c|c|}
\hline \multirow[b]{2}{*}{ Compd. } & \multicolumn{2}{|c|}{$\lambda_{\text {test }} / \lambda_{\text {exc }} / \mathrm{nm}$} & \multicolumn{2}{|c|}{$\tau_{\mathrm{m}} / \mu \mathrm{s}$} & \multicolumn{2}{|c|}{$\tau_{\mathrm{o}} / \mu \mathrm{s}$} & \multicolumn{2}{|c|}{$k_{\mathrm{SS}} / \times 10^{6} \mathrm{~s}^{-1}$} & \multicolumn{2}{|l|}{$\Phi^{\prime}$} & \multicolumn{2}{|c|}{$\Phi_{\mathrm{ISC}}($ calc. $)$} \\
\hline & $\mathrm{H}_{2}$ & $\mathrm{Zn}$ & $\mathrm{H}_{2}$ & $\mathrm{Zn}$ & $\mathrm{H}_{2}$ & $\mathrm{Zn}$ & $\mathrm{H}_{2}$ & $\mathrm{Zn}$ & $\mathrm{H}_{2}$ & $\mathrm{Zn}$ & $\mathrm{H}_{2}$ & $\mathrm{Zn}$ \\
\hline TPP & $442 / 524$ & $480 / 565$ & 0.48 & 0.7 & 385 & 815 & 2.1 & 1.3 & 1 & 1 & 0.65 & 0.75 \\
\hline DETPP & $454 / 524$ & $480 / 565$ & 0.43 & 0.7 & 13.6 & 25.2 & 2.3 & 1.4 & 0.97 & 0.97 & 0.63 & 0.63 \\
\hline tTETPP & $474 / 524$ & $480 / 565$ & 0.66 & 0.8 & 21.7 & 20 & 1.5 & 1.07 & 0.97 & 0.96 & 0.56 & 0.35 \\
\hline cTETPP & $514 / 608$ & $490 / 565$ & 0.27 & 0.7 & 0.42 & 1.41 & 1.3 & 0.61 & 0.36 & 0.46 & 0.22 & 0.2 \\
\hline НЕTPP & $514 / 608$ & - & 0.45 & - & 0.9 & - & 1.1 & - & 0.5 & - & 0.14 & - \\
\hline ОЕTPP & $514 / 608$ & $514 / 590$ & 1.1 & 0.5 & 5.4 & 1.88 & 0.72 & 1.35 & 0.8 & 0.72 & 0.25 & 0.25 \\
\hline
\end{tabular}




\section{Experimental}

\section{Synthesis and structural analysis of compounds}

Compounds were synthesized as described earlier. ${ }^{16}$ Structural parameters were derived from the crystallographic data reported earlier using standard porphyrin conformational analyses ${ }^{7,25}$ and normal structural decomposition analyses. ${ }^{26}$

\section{Spectroscopic methods}

The absorption spectra were recorded using a commercial spectrophotometer Shimadzu UV160A. All optical investigations were carried out in DMF or MTHF.

Time resolved fluorescence measurements were carried out using time-correlated single photon counting (TCSPC), containing an SPC 300 plug-in card (Becker Hickl, Berlin) and a multi-channel plate (Hamamatsu) as described earlier. ${ }^{23}$ For data analysis the decay curves were deconvoluted and fitted using the least-squares method based on a Newton algorithm. For monoexponential fits $\chi^{2}$-values less than 1.1 were reached. For fittings with more than one decay time, even smaller $\chi^{2}$-values close to 1.0 were obtained.

The device for steady-state luminescence measurements was based on an L-setup with different light sources (Xe lamp, Yb+ Disc-YAG pumped dye laser) and has been described previously. ${ }^{27}$

The singlet oxygen quantum yield $\left(\Phi_{\Delta}\right)$ of all samples was determined twice using steady state (nitrogen cooled InGaAsdiode, Laser Components) and time resolved (nitrogen cooled Ge-diode, Northcoast and recording oscilloscope HP) luminescence detection $^{28}$ to exclude the influence of different possible non-radiative deactivation pathways. Using these two different methods identical quantum yields $\left(\Phi_{\Delta} \pm 0.05\right)$ were obtained for all samples. For this reason only one value of $\Phi_{\Delta}$ is given for each sample in the results section. The temporal shape of singlet oxygen luminescence in a homogeneous environment can be determined using the following eqn (2): ${ }^{29}$

$$
I_{\Delta}=N \Phi_{\Delta}\left[\exp \left(-\frac{t}{\tau_{\Delta}}\right)-\exp \left(-\frac{t}{\tau_{\mathrm{T}}}\right)\right] \frac{\tau_{\Delta}}{\tau_{\Delta}-\tau_{\mathrm{T}}}
$$

where $I_{\Delta}$ is the singlet oxygen luminescence intensity. $N$ is the population of excited photosensitizer molecules, which is determined by the light excitation intensity, the photosensitizer absorption at excitation wavelength and the photosensitizer concentration. $\Phi_{\Delta}$ is the singlet oxygen quantum yield, which changes with the photosensitizer, and different other parameters like the quencher concentration, the oxygen concentration, the solvent. $\tau_{\Delta}$ is the singlet oxygen lifetime and $\tau_{\mathrm{T}}$ is the triplet lifetime of the photosensitizer.

The singlet oxygen luminescence decay curves obtained were fitted with the two-exponential model function (eqn (2)). As can be seen, the shape of the singlet oxygen luminescence is determined by two competitive lifetimes: the triplet lifetime of photosensitizer $\tau_{\mathrm{T}}$ and the singlet oxygen lifetime $\tau_{\Delta}$. The rise of the singlet oxygen luminescence is determined by the faster one. The slower one determines the signal decay. The triplet lifetime of the photosensitizer in solution is usually shorter than the singlet oxygen lifetime. Therefore, the rising signal presents the triplet lifetime $\tau_{\mathrm{T}}$. The decay signal is due to the singlet oxygen lifetime $\tau_{\Delta}$.
Laser flash photolysis. The same equipment was used for triplettriplet absorption measurements as for the time resolved singlet oxygen luminescence detection. The triplet lifetime of a photosensitizer can be calculated by analyzing the transient absorption curve. A continuous wave (cw) test beam was generated using an XBO lamp with the light passed through a monochromator. The beam traversed through the solutions perpendicular to the excitation beam and was detected by an avalanche Si-photodiode with an interference filter at $480 \mathrm{~nm}$.

Transient absorption measurements (ps-TAS) for the determination of intersystem crossing quantum yields $\left(\Phi_{\text {ISC }}\right)$ were performed using a pump-probe-setup described earlier. ${ }^{30}$

\section{Conclusions}

The data presented here clearly show that a conformationally designed series of porphyrins with graded degree of macrocycle distortion exhibits gradually altered photophysical properties. This further indicates the possibility to use such systems for the preparation of biomimetic and catalytically active chromophores with fine-tunes photophysical parameters.

\section{Acknowledgements}

This work (M.S.) was generously supported by grants from Science Foundation Ireland (Research Professorship Award SFI 04/RP1/B482 and P.I. 09/IN.1/B2650).

\section{Notes and references}

1 L. R. Milgrom, The Colours of Life, Oxford University Press, Oxford, 1987.

2 M. O. Senge, J. Photochem. Photobiol., B, 1992, 16, 3-36.

3 J. A. Shelnutt, X. Z. Song, J. G. Ma, S. L. Jia, W. Jentzen and C. J. Medforth, Chem. Soc. Rev., 1998, 27, 31-41.

4 A. Forman, M. W. Renner, E. Fujita, K. M. Barkigia, M. C. W. Evans, K. M. Smith and J. Fajer, Isr. J. Chem., 1989, 29, 57-64.

5 R. Huber, Eur. J. Biochem., 1990, 187, 283-305.

6 K. M. Barkigia, M. D. Berber, J. Fajer, C. J. Medforth, M. W. Renner and K. M. Smith, J. Am. Chem. Soc., 1990, 112, 8851-8857.

7 M. O. Senge, in The Porphyrin Handbook, ed. K. M. Kadish, K. M. Smith and R. Guilard, Academic Press, San Diego, 1990, vol. 1, pp. 239-347.

8 M. O. Senge, Chem. Commun., 2006, 243-256.

9 Z. Shi, R. Franco, R. Haddad, J. A. Shelnutt and G. C. Ferreoira, Biochemistry, 2006, 45, 2904-2912.

10 L. C. Gong and D. Dolphin, Can. J. Chem., 1985, 63, 401-405.

11 M. B. Hursthouse and S. Neidle, J. Chem. Soc., Chem. Commun., 1972, 449-450.

12 J.-H. Fuhrhop, L. Witte and W. S. Sheldrick, Justus Liebigs Ann. Chem., 1976, 1537-1559.

13 C. J. Medforth, M. O. Senge, K. M. Smith, L. D. Sparks and J. A. Shelnutt, J. Am. Chem. Soc., 1992, 114, 9859-9869.

14 H. Ogura, L. Yatsunyk, C. J. Medforth, K. M. Smith, K. M. Barkigia, M. W. Renner, D. Melamed and F. A. Walker, J. Am. Chem. Soc., 2001, 123, 6564-6578; R. Weiss, J. Fischer, V. Bulach, V. Schunemann, M. Gerdan, A. X. Trautwein, J. A. Shelnutt, C. P. Gros, A. Tabard and R. Guilard, Inorg. Chim. Acta, 2002, 337, 223-232; L. A. Yatsunyk, N. V. Shokhirev and F. A. Walker, Inorg. Chem., 2005, 44, 2848-2866; L. A. Poveda, V. R. Ferro, J. M. G. de la Vega and R. H. Gonzalez-Jonte, Phys. Chem. Chem. Phys, 2000, 2, 4147-4156; Y. Ohgo, A. Hoshino, T. Okamura, H. Uekusa, D. Hashizume, A. Ikezaki and M. Nakamura, Inorg. Chem., 2007, 46, 8193-8207.

15 J. L. Retsek, S. Gentemann, C. J. Medforth, K. M. Smith, V. S. Chirvony, J. Fajer and D. Holten, J. Phys. Chem. B, 2000, 104, 6690-6693; S. Gentemann, C. J. Medforth, T. P. Forsyth, D. J. Nurco, K. M. Smith, J. Fajer and D. Holten, J. Am. Chem. Soc., 1994, 116, 7363-7368. 
16 W. W. Kalisch and M. O. Senge, Tetrahedron Lett., 1996, 37, 1183-1186; M. O. Senge and W. W. Kalisch, Inorg. Chem., 1997, 36, 6103-6116.

17 S. Michaeli, S. Soffer, H. Levanon, M. O. Senge and W. W. Kalisch, J. Phys. Chem. A, 1999, 103, 1950-1957.

18 M. O. Senge, W. W. Kalisch and S. Runge, Liebigs Ann./Recl., 1997, 1345-1352; M. O. Senge and W. W. Kalisch, Z. Naturforsch, 1999, 54b, 943-959; R. Weiss, J. Fischer, V. Bulach and J. A. Shelnutt, C. R. Chim., $2002,5,405-416$

19 M. O. Senge, W. W. Kalisch and S. Runge, Tetrahedron, 1998, 54, 37813798; M. O. Senge, V. Gerstung, K. Ruhlandt-Senge, S. Runge and I. Lehmann, J. Chem. Soc., Dalton Trans., 1998, 4187-4199.

20 I. V. Sazanovich, V. A. Galievsky, A. van Hoek, T. J. Schaafsma, V. L. Malinosvskii, D. Holten and V. S. Chirvony, J. Phys. Chem. B, 2001, 105, 7818-7829.

21 V. S. Chirvony, I. V. Avilov, A. Y. Panarin, V. L. Malinovskii and V. A. Galievsky, Chem. Phys. Lett., 2007, 434, 116-120.

22 C. J. Medforth, M. D. Berber, K. M. Smith and J. A. Shelnutt, Tetrahedron Lett., 1990, 31, 3719-3722.
23 J. Zimmermann, J. v. Gersdorff, H. Kurreck and B. Röder, $J$. Photochem. Photobiol., B, 1997, 40, 209-217.

24 A. A. Krasnowski Jr., Proc. Royal Soc. Edinburgh, 1992, 102B, 219

25 W. R. Scheidt and Y. J. Lee, Struct. Bonding, 1987, 64, 1-70.

26 W. Jentzen, X.-Z. Song and J. A. Shelnutt, J. Phys. Chem. B, 1997, 101, 1684-1699; W. Jentzen, J.-G. Ma and J. A. Shelnutt, Biophys. J., 1998, 74, 753-763.

27 O. Korth, T. Hanke, I. Rückmann and B. Rö der, Exp. Tech. Phys., 1995, 41, 25-36.

28 S. Oelckers, M. Sczepan, T. Hanke and B. Röder, J. Photochem. Photobiol., B, 1997, 39, 219-223; W. Spiller, H. Kliesch, D. Wöhrle, S. Hackbarth and B. Röder, J. Porphyrins Phthalocyanines, 1998, 2, $145-158$.

29 J. Schlothauer, S. Hackbarth and B. Röder, Laser Phys. Lett., 2009, 6, $169-174$.

30 A. Zeug, I. Rückmann and B. Röder, J. Opt. B: Quantum Semiclassical Opt., 2001, 3, S251-S258. 